\title{
GENOMICS
}

\section{PAM-less is more}

\author{
Structure-guided engineering allows researchers access to an expanding number of Cas9 variants that relax the \\ constraint imposed by target site recognition of a protospacer-adjacent motif (PAM).
}

$\mathrm{N}$ aturally occurring CRISPR enzymes recognize their targets with the help of guide RNAs, and this target recognition is constrained by the need for a PAM sequence. For example, the well-known Streptococcus pyogenes Cas9 (SpCas9) requires target recognition of the NGG motif, where $\mathrm{N}$ can be $\mathrm{A}, \mathrm{C}, \mathrm{G}$, or $\mathrm{T}$. In the context of genome editing, the requirement to recognize PAM reduces CRISPR targeting resolution and leaves some genome sites inaccessible to editing.

To allow CRISPR enzymes access more of the genome, researchers have applied bioinformatics-assisted ortholog discovery and protein engineering to identify Cas proteins with different PAM preferences. Previously, the Joung and Kleinstiver groups from Massachusetts General Hospital successfully used directed evolution to alter the PAM requirements of SpCas9 from NGG to NGA, and also altered the Staphylococcus aureus Cas9 (SaCas9) PAM from NNGRRN to NNNRRT (where R is A or G). But one question remained, as Kleinstiver notes: "Is recognition of a specific PAM by CRISPR enzymes truly required for Cas proteins to target DNA?"

To further expand the number of PAMs accessible to CRISPR enzymes, structure-guided engineering can be used to generate additional Cas variants. Directed evolution and structure-guided engineering are two common routes for engineering enzyme variants; each holds advantages and limitations. Directed evolution can be advantageous when little is known about the enzyme's biochemical properties and structure. Yet the inability to generate complex and saturating libraries may hinder sampling of the entire mutagenesis space. Conversely, structure-guided engineering mutagenizes fewer residues but at a greater depth, using structural information as a rational guide for which residues to test.

Regarding the choice of engineering approach, Kleinstiver comments, "For Cas proteins where structural information is available, there is now a clear blueprint for relaxing the PAM requirement. For Cas orthologs for which structural information is not available, or where high-quality protein alignments or homology models

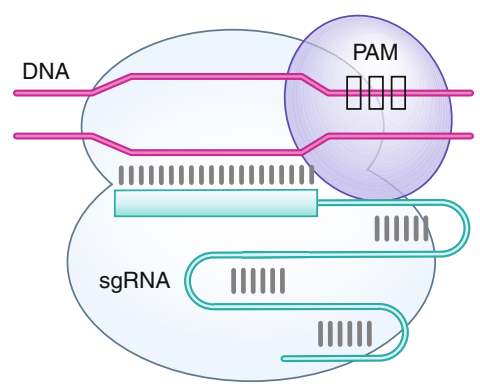

CRISPR enzymes require a PAM sequence for target site recognition.

cannot be generated, then structure-guided engineering is more challenging."

Guided by the SpCas9 structural information and the information obtained when generating previous SpCas9 variants, Kleinstiver and colleagues hypothesized that amino acid substitutions in the PAM-interacting domain of SpCas9 would make it possible to unconstrain PAM preferences (see Walton et al.). To facilitate the characterization of many SpCas9 variants, they also developed a high-throughput PAM determination assay (HT-PAMDA) to assess the PAM requirements of various SpCas9 variants containing rational substitutions at key residues.

Among the variants, they identified an enzyme they call SpG that exhibits a more relaxed PAM requirement of NGN. Further substitutions at critical PAM-interacting positions allowed them to generate a near-PAM-less SpCas9 variant named SpRY. In HEK293T cells, SpRY exhibits comparable editing activity at recognition sites with NRN PAMs and at 13 out of 31 recognition sites with NYN PAMs (where $\mathrm{Y}$ is $\mathrm{C}$ or $\mathrm{T}$ ).

In addition to nuclease targeting, the researchers also evaluated the capabilities of SpG and SpRY in the context of a cytosine base editor (CBE) or adenine base editor (ABE), to better position the edit windows of the base editors using PAMs beyond NGG. To showcase the utility of these new base editors, which can target an expanded number of target sites, SpG- and SpRY-CBEs were used to generate human-disease-relevant $\mathrm{C}$-to-T edits that were inaccessible to the wild-type CBE. Moreover, the availability of more target sites circumvents the unwanted editing of bystander cytosines.

DNA targeting specificity is another critical factor in genome editing. "Since the PAM requirement is a major determinant of DNA-targeting specificity, it stands to reason that enzymes with truly relaxed PAMs should be able to edit additional off-target sites," says Kleinstiver. Not surprisingly, GUIDE-seq experiments indicated that SpG and SpRY are able to edit additional off-target sites because of the expanded PAMs. The Kleinstiver team sought to regain target specificity by engineering fidelity-enhancing substitutions into the SpG and SpRY PAM variants. The dual protein engineering is possible "since PAM recognition and substrate proofreading are in principle distinct properties," Kleinstiver says. Their results suggest that high-fidelity variants of SpG and SpRY eliminate the majority of the off-target editing events.

In an independent study, Chatterjee et al. from the Massachusetts Institute of Technology leveraged evolutionary information from Streptococcus Cas9 orthologs to engineer a high-fidelity variant of Streptococcus canis Cas9 (ScCas9) that exhibits improved specificity with an expanded PAM of NNG. They also demonstrated that mutations introduced into SpCas9, such as mutations that increase fidelity, may also be applicable to engineering ScCas9 variants.

With these PAM-less variants in hand, there remain questions to be answered. "Whether or not the relaxed PAM enzymes behave similarly in terms of substrate recognition and requirements is an open question that merits further investigation," Kleinstiver says.

\section{Lei Tang}

Published online: 4 June 2020

https://doi.org/10.1038/s41592-020-0861-5

\section{Research papers}

Walton, R. T. et al. Unconstrained genome targeting with near-PAMless engineered CRISPR-Cas9 variants. Science 368, 290-296 (2020).

Chatterjee, P. et al. An engineered ScCas9 with broad PAM range and high specificity and activity. Nat. Biotechnol. https://doi.org/10.1038/s41587-0200517-0 (2020). 